\title{
On embedding of the Bratteli diagram into a surface
}

\author{
Igor Nikolaev \\ Department of Mathematics \\ 2500 University Drive N.W. \\ Calgary T2N 1N4 Canada \\ nikolaev@math.ucalgary.ca
}

November 4, 2018

\begin{abstract}
We study $C^{*}$-algebras $\mathcal{O}_{\lambda}$ which arise in dynamics of the interval exchange transformations and measured foliations on compact surfaces. Using Koebe-Morse coding of geodesic lines, we establish a bijection between Bratteli diagrams of such algebras and measured foliations. This approach allows us to apply $K$-theory of operator algebras to prove strict ergodicity criterion and Keane's conjecture for the interval exchange transformations.
\end{abstract}

Key words and phrases: K-theory, dimension group, measured foliation

AMS (MOS) Subj. Class.: 19K14, 46L40, 57R30.

\section{Introduction}

Let $\lambda=\left(\lambda_{1}, \ldots, \lambda_{n}\right)$ be a partition of the unit interval into a disjoint union of open subintervals. Let $\varphi:[0,1] \rightarrow[0,1]$ be an interval exchange transformation (with flips). Consider a unital $C^{*}$-algebra $\mathcal{O}_{\lambda}$ generated by the unitary operator $u(\zeta)=\zeta \circ \varphi^{-1}$ and characteristic operators $\chi_{\lambda_{1}}, \ldots, \chi_{\lambda_{n}}$ 
in the Hilbert space $L^{2}([0,1])$. This (noncommutative) $C^{*}$-algebra has an amazingly rich geometry.

$\mathcal{O}_{\lambda}$ is Morita equivalent to a groupoid $C^{*}$-algebra corresponding to measured foliations on a compact surface of genus greater than one. To this end, $\mathcal{O}_{\lambda}$ is an extension of the irrational rotation algebra $A_{\theta}$ whose theory experienced an extensive development in the last decades.

$\mathcal{O}_{\lambda}$ is closely related to simple $C^{*}$-algebras of minimal homeomorphisms on a Cantor set. These $C^{*}$-algebras were in focus of a brilliant series of works of I. F. Putnam starting with the papers [10], [11]. We refer the reader to our work [9] for discussion of connections between Putnam's algebras and $\mathcal{O}_{\lambda}$.

The $K$-groups of $\mathcal{O}_{\lambda}$ are finitely generated and can be obtained from the Pimsner-Voiculescu diagram for the crossed products. Namely, $K_{0}\left(\mathcal{O}_{\lambda}\right)=\mathbb{Z}^{n}$, $K_{1}\left(\mathcal{O}_{\lambda}\right)=\mathbb{Z}$, where $n$ is the number of intervals in the partition of $[0,1]$. The dimension group $\left(K_{0}, K_{0}^{+},[1]\right)$ of $\mathcal{O}_{\lambda}$ was calculated in [9]. (The reader is referred to Appendix for the details of this construction.) When $\varphi$ is minimal the dimension group $\left(K_{0}, K_{0}^{+},[1]\right)$ is simple.

Recall that state on dimension group is a positive homomorphism of $\left(K_{0}, K_{0}^{+},[1]\right)$ to $\mathbb{R}$ which respects the order units [1] and $1 \in \mathbb{R}$. The state space $S_{\bullet}$ of $\left(K_{0}, K_{0}^{+},[1]\right)$ is a Choquet simplex of dimension $\leq n-1$. The dimension of $S_{\bullet}$ is equal to the number of linearly independent invariant ergodic measures of $\varphi$. Each invariant measure corresponds to a 1-dimensional linear subspace of the state space and $\operatorname{dim} S_{\bullet}=1$ if and only if the interval exchange transformation $\varphi$ is strictly (uniquely) ergodic.

It might be one of the most intriguing problems of topological dynamics since 25 years to indicate conditions of strict ergodicity of $\varphi$. Some results in this direction are due to Veech and Boshernitzan. In 1975 Keane conjectured that "typical" $\varphi$ is strictly ergodic. Masur [6] and Veech [15] proved this conjecture in positive using methods of complex analysis and topological dynamics, respectively.

This note is an attempt to study dynamics of $\varphi$ using the ideas and methods of operator algebras. A foundation to such an approach is given by the following main theorem (to be proved in Section 2):

Theorem 1 Let $n \geq 2$ be an integer. Let $\left(P, P_{+},[u]\right)$ be a simple and totally ordered 1 dimension group of order $n \geq 2$. Then there exists an interval

\footnotetext{
${ }^{1}$ Total ordering condition ensures that the Unimodular Conjecture is true, see Effros 2 and Elliott [3]. The author believes the condition is technical, but cannot drop it at this stage.
} 
exchange transformation $\varphi=\varphi(\lambda, \pi, \varepsilon)$ of $n$ intervals and a $C^{*}$-algebra $\mathcal{O}_{\lambda}$ with the group $\left(K_{0}, K_{0}^{+},[1]\right)$ which is order-isomorphic to $\left(P, P_{+},[u]\right)$. The transformation $\varphi$ is minimal.

The proof of the above theorem is based on the identification of the infinite paths of Bratteli diagram with the symbolic geodesics on a compact surface (so-called Koebe-Morse theory). This method has an independent interest since it provides direct links between geometry of geodesics and $K$-theory of operator algebras.

The paper is divided into five sections. In Section 1 we introduce notation and a lemma on positive cone in $K_{0}\left(\mathcal{O}_{\lambda}\right)$. In Section 2 we give the proof of main theorem. In Sections 3 and 4 we apply Theorem 1 to establish a strict ergodicity criterion and Keane's Conjecture, respectively. Section 5 is an Appendix containing quick review of dynamics of the interval exchanges, measured foliations, $K$-theory and rotation numbers associated to the $C^{*}$-algebra $\mathcal{O}_{\lambda}$. The reader is encouraged to read "Conclusions and open problems" section at the end of this paper.

Acknowledgements. I wish to thank G. A. Elliott for many helpful discussions and ideas.

\section{Notations}

Let $A$ be a unital $C^{*}$-algebra and $V(A)$ be the union (over $n$ ) of projections in the $n \times n$ matrix $C^{*}$-algebra with entries in $A$. Projections $p, q \in V(A)$ are equivalent if there exists a partial isometry $u$ such that $p=u^{*} u$ and $q=u u^{*}$. The equivalence class of projection $p$ is denoted by $[p]$.

Equivalence classes of orthogonal projections can be made to a semigroup by putting $[p]+[q]=[p+q]$. The Grothendieck completion of this semigroup to an abelian group is called a $K_{0}$-group of algebra $A$.

Functor $A \rightarrow K_{0}(A)$ maps a category of unital $C^{*}$-algebras into the category of abelian groups so that projections in algebra $A$ correspond to a "positive cone" $K_{0}^{+} \subset K_{0}(A)$ and the unit element $1 \in A$ corresponds to an "order unit" [1] $\in K_{0}(A)$. The ordered abelian group $\left(K_{0}, K_{0}^{+},[1]\right)$ with an order unit is called a dimension (Elliott) group of $C^{*}$-algebra $A$.

For the $C^{*}$-algebra $\mathcal{O}_{\lambda}$ one easily finds that $K_{0}\left(\mathcal{O}_{\lambda}\right)=\mathbb{Z}^{n}$, see the Appendix. It is harder to figure out the positive cone $K_{0}^{+}\left(\mathcal{O}_{\lambda}\right)$. The rest of the section is devoted to this specific question. 
Let us fix the following notation:

$\mathbb{H} \quad$ Lobachevsky complex half-plane $\{z=x+i y \mid y>0\}$ endowed with the hyperbolic metric $d s=|d z| / y$;

$\partial \mathbb{H} \quad$ absolute, i.e. line $y=0$ of the Lobachevsky half-plane;

G $\quad$ Fuchsian group of the first kind;

$M_{g, m} \quad$ orientable surface of genus $g$ with $m$ boundary components;

$\mathcal{F} \quad$ measured foliation of $M_{n}$ obtained as suspension over interval exchange transformation $\varphi=\varphi(\lambda, \pi, \varepsilon)$ with $n$ intervals;

$\Lambda \quad$ geodesic lamination corresponding to $\mathcal{F}$.

$\gamma \quad$ geodesic "generating" $\Lambda$, i.e. $\bar{\gamma}=\Lambda$.

Thurston has shown that each measured foliation $\mathcal{F}$ can be represented by a "geodesic lamination" $\Lambda$ consisting of disjoint non-periodic geodesics, which lie in the closure of any one of them; cf Thurston [13]. Denote by $p: \mathbb{H} \rightarrow$ $M_{g, m}$ a covering mapping corresponding to the action of discrete group $G$.

The geodesic lamination $\Lambda$ is a product $K \times \mathbb{R} \subset M_{g, m}$, where $K$ is a (linear) Cantor set. The preimage $p^{-1}(\Lambda) \subset \mathbb{H}$ is a collection of geodesic half-circles without self-intersections except, possibly, at the absolute. The "footpoints" of these half-circles is a subset of $\partial \mathbb{H}$ homeomorphic to $K$.

Fix a Riemann surface $M_{g, m}=\mathbb{H} / G$ of genus $g$ together with a point $p \in M_{g, m}$. Let $\gamma$ be a "generating" geodesic of the lamination $\Lambda$, i.e. such that closure $\bar{\gamma}=\Lambda$. Consider the set

$$
S p(\gamma)=\left\{\gamma_{0}, \gamma_{1}, \gamma_{2}, \ldots\right\}
$$

of periodic geodesics $\gamma_{i}$ based in $p$, which monotonically approximate $\gamma$ in terms of "length" and "direction". The set $S p(\gamma)$ is known as spectrum of $\gamma$ and is defined uniquely upon $\gamma$.

Let $n=2 g+m-1$. Then the (relative) integral homology $H_{1}\left(M_{g, m}, \partial M_{g, m} ; \mathbb{Z}\right) \cong$ $\mathbb{Z}^{n}$. Since each $\gamma_{i}$ is a 1-cycle, there is an injective map $f: S p(\gamma) \rightarrow$ $H_{1}\left(M_{g, m}, \partial M_{g, m} ; \mathbb{Z}\right)$, which relates every closed geodesic its homology class. 
Note that $f\left(\gamma_{i}\right)=p_{i} \in \mathbb{Z}^{n}$ is "prime" in the sense that it is not an integer multiple of some other point of lattice $\mathbb{Z}^{n}$. Denote by $S p_{f}(\gamma)$ the image of $S p(\gamma)$ under mapping $f$. Finally, let $S L(n, \mathbb{Z})$ be the group of $n \times n$ integral matrices of determinant 1 and $S L\left(n, \mathbb{Z}^{+}\right)$its semigroup consisting of matrices with strictly positive entries. It is not hard to show, that in appropriate basis in $H_{1}\left(M_{g, m}, \partial M_{g, m} ; \mathbb{Z}\right)$ the following is true:

(i) the coordinates of vectors $p_{i}$ are non-negative;

(ii) there exists a matrix $A_{i} \in S L\left(n, \mathbb{Z}^{+}\right)$such that $p_{i}=A_{i}\left(p_{i-1}\right)$ for any pair of vectors $p_{i-1}, p_{i}$ in $S p_{f}(\gamma)$.

Definition 1 The ordered abelian group $\left(\mathbb{Z}^{n},\left(\mathbb{Z}^{n}\right)^{+},[1]\right)$ defined as inductive limit of simplicially ordered groups:

$$
\mathbb{Z}^{n} \stackrel{\mathrm{A}_{1}}{\longrightarrow} \mathbb{Z}^{n} \stackrel{\mathrm{A}_{2}}{\longrightarrow} \mathbb{Z}^{n} \stackrel{\mathrm{A}_{3}}{\longrightarrow} \ldots
$$

is called associated to geodesic $\gamma$.

(We have shown in [9] that the order structure on $\left(\mathbb{Z}^{n},\left(\mathbb{Z}^{n}\right)^{+},[1]\right)$ is independent of the choice of $M_{g, m}$ and $\gamma$.)

Lemma 1 dimension group $\left(K_{0}, K_{0}^{+},[1]\right)$ of the $C^{*}$-algebra $\mathcal{O}_{\lambda}$ is orderisomorphic to the associated group $\left(\mathbb{Z}^{n},\left(\mathbb{Z}^{n}\right)^{+},[1]\right)$ of Definition 1 .

Proof. See [9].

\section{Proof of Theorem 1}

Let us outline main idea of the proof. To every dimension group $\left(P, P_{+},[u]\right)$ with $P \simeq \mathbb{Z}^{n}$ one can relate a Bratteli diagram $(V, E)$. The path space $X$ of $(V, E)$ can be made to a topological space by putting two paths "close" if and only if they coincide at the initial steps. ( $X$ is called Bratteli-Cantor compactum.) $X$ can be embedded (as topological space) into the complex plane $\mathbb{H}$ by identification of each $x \in X$ with a geodesic in $\mathbb{H}$ via Morse coding of the geodesic lines. We show that $X=p^{-1}(\Lambda)$, where $\Lambda$ is Thurston's geodesic lamination on the surface $M_{n}=\mathbb{H} / G$; cf Thurston [13]. A concluding step is to recover $\mathcal{F}$ and $\varphi$ from $\Lambda$.

Let $\left(P, P_{+},[u]\right)$ be a simple totally ordered dimension group with $P \simeq \mathbb{Z}^{n}$. Recall that a Bratteli diagram of $\left(P, P_{+},[u]\right)$ consists of a vertex set $V$ and 
edge set $E$ such that $V$ is an infinite disjoint union $V_{1} \sqcup V_{2} \sqcup \ldots$, where each $V_{i}$ has cardinality $n$. The latter condition follows from the total ordering of $\mathbb{Z}^{n}$. Any pair $V_{i-1}, V_{i}$ defines a non-empty set $E_{i} \subset E$ of edges with a pair of range and source functions $r, s$ such that $r\left(E_{i}\right) \subseteq V_{i}$ and $s\left(E_{i}\right) \subseteq V_{i-1}$.

An $A F C^{*}$-algebra whose dimension group is order-isomorphic to $\left(P, P_{+},[u]\right)$ is an inductive limit of multi-matrix algebras

$$
\lim M_{J_{1}}(\mathbb{C}) \oplus \ldots \oplus M_{J_{n}}(\mathbb{C})
$$

We shall say that a Bratteli diagram $(V, E)$ corresponds to group $\left(P, P_{+},[u]\right)$ if the range and source functions of $(V, E)$ represent embedding scheme of the above multi-matrix algebras. (In other words, an $A F$-algebra defined by $(V, E)$ has Elliott group $\left(P, P_{+},[u]\right)$.)

The equivalence class of Bratteli diagrams corresponding to a simple totally ordered dimension group of form $\mathbb{Z}^{n}$ has a representative $(V, E)$ with no multiple edges, since every positive integral matrix decomposes into a finite product of non-negative matrices whose entries are zeros and ones. For the sake of simplicity, we always assume this case of Bratteli diagrams.

By an infinite path on $(V, E)$ we shall mean an infinite sequence of edges $\left(e_{0}, e_{1}, \ldots\right)$ such that $e_{0} \in E_{0}, e_{1} \in E_{1}$, etc. The set of all infinite paths on $(V, E)$ is denoted by $X$. Let us identify "coordinates" $x_{i}$ of $x \in X$ with vector $\left(e_{0}, e_{1}, \ldots\right)$. Fix $x, y \in X$. Metric $d(x, y)=1 / 2^{k}$, where

$$
k=\max \left\{l \in \mathbb{N} \mid x_{i}=y_{i} \text { for } i<l\right\},
$$

turns $X$ into an absolutely disconnected topological space which is called a Bratteli-Cantor compactum. To construct an embedding $X \rightarrow \mathbb{H}$ where each $x \in X$ represents a geodesic, a portion of symbolic dynamics is needed.

Koebe-Morse coding of geodesics. Let $M_{g, m}$ be a hyperbolic surface of genus $g$ with $m$ totally geodesic boundary components $v_{1}, \ldots, v_{m}$. We dissect $M_{g, m}$ to a simply connected surface as follows [7]. Let $P$ be an arbitrary point of $v_{m}$. One draws geodesic segments $h_{1}, \ldots, h_{m-1}$ from $P$ to some arbitrary chosen points of $v_{1}, \ldots, v_{m-1}$. (Thus, $h_{i}$ have only $P$ as common point.) Next one dissects the handles of $M_{g, m}$ by closed geodesics $c_{1}, \ldots, c_{2 g}$ issued from point $P$. Clearly, the resulting surface is simply connected and has the boundary

$$
c_{1}, \ldots, c_{2 g} ; h_{1}, \ldots, h_{m-1} \text {. }
$$


Now given geodesic half-circle $S \subset \mathbb{H}$ passing through unique point $0 \in \tau$ one relates an infinite sequence of symbols

$$
\sigma_{1}, \sigma_{2}, \sigma_{3}, \ldots
$$

which "take values" in the set $\sigma$. One prescribes $\sigma_{p}, p=1, \ldots, \infty$ a "value" $g_{i}, 1 \leq i \leq n$ if and only if $S$ has a transversal intersection point with the side $a_{i}=b_{i}$ of $p$-th image of $G_{\sigma} \in \tau$. (In other words, code (4) "counts" points of intersection of $S$ with "sides" of tessellation $\tau$.) Sequence of symbols (4) is called a Koebe-Morse code of geodesic $S$.

Morse showed that there is a bijective correspondence between sequences (4) satisfying some admissibility requirements 2 and the set of non-periodic geodesics on surfaces of negative curvature; see the bibliography to Morse and Hedlund [8].

Lemma 2 Let $S$ be a geodesic with the Koebe-Morse code $\left(\sigma_{1}, \sigma_{2}, \ldots\right)$. Then any congruent to $S$ geodesic $S^{\prime}$ will have the same Koebe-Morse code, except possibly in a finite number of terms.

Proof. This follows from the definition of coding and invariance of $\tau$ by the $G$-actions.

Let $X$ be a Bratteli-Cantor compactum. Let $V_{i} \rightarrow \sigma$ be a bijection between the vertices $V=V_{1} \sqcup V_{2} \sqcup \ldots$ of $(V, E)$ and the set of symbols $\sigma$. This bijection can be established by labeling each element of $V_{i}$ from the left to the right by symbols $\left\{g_{1}, \ldots, g_{n}\right\}$. Thus, every $x \in X$ is a "symbolic geodesic" $\left(x_{1}, x_{2}, \ldots\right)$ whose "coordinates" take values in $\sigma$. Each sequence is admissible and by Morse's Theorem realized by a (class of congruent) geodesic whose KoebeMorse code coincides with $\left(x_{1}, x_{2}, \ldots\right)$. Where there is no confusion, we refer to $x \in X$ as a geodesic line in the complex plane $\mathbb{H}$.

Lemma 3 Let $l_{x}$ be an image of geodesic $x \in X$ on the surface $M_{g, m}$ under projection $\mathbb{H} \rightarrow \mathbb{H} / G$. If Bratteli diagram $(V, E)$ is simple, then $l_{y} \in C l o s l_{x}$ for any $y \in X$.

Proof. The simplicity of $(V, E)$ means that every infinite path $x \in X$ is transitive, i.e. any finite "block" of symbols $\left\{x_{n}, x_{n+1}, \ldots, x_{n+k}\right\}$ occurs "infinitely many times" in the sequence $x=\left(x_{1}, x_{2}, \ldots\right)$. Indeed, simplicity of

\footnotetext{
${ }^{2}$ Namely, there should be no words with the syllabi $a_{i} b_{i}$ or $b_{i} a_{i}$, where $a_{i}$ and $b_{i}$ are "dual" symbols from the alphabet $G_{\sigma}$.
} 
$(V, E)$ means the absence of non-trivial ideals in the corresponding $A F C^{*}$ algebra. Using Bratteli's dictionary (1]) between ideals and connectedness properties of $(V, E)$, it can be easily shown that arbitrary infinite path in $(V, E)$ "visits" any given finite sequence of vertices infinitely often.

Suppose that $B_{k}$ is a block of symbols of length $k \geq 1$. Let

$$
x=\left(x_{1}, \ldots, x_{n-1}, B_{k}, x_{n+k+1}, \ldots\right), \quad y=\left(y_{1}, \ldots, y_{m-1}, B_{k}, y_{m+k+1}, \ldots\right),
$$

be the first time $B_{k}$ appears in sequences $x, y \in X$. By a congruent transformation, the geodesics $x, y \in \mathbb{H}$ can be brought to the from

$$
x^{\prime}=\left(B_{k}, x_{k+1}, \ldots\right), \quad y^{\prime}=\left(B_{k}, y_{k+1}, \ldots\right) .
$$

This means that dist $\left(x^{\prime}, y^{\prime}\right) \leq 1 / 2^{k}$. Since $B_{k}$ occurs in sequences $x$ and $y$ infinitely often, lemma follows.

Lemma 4 The set Clos $l_{x}$ of Lemma 3 is a set $\Lambda \subset M_{g, m}$ consisting of continuum of irrational geodesic lines.

Proof. This follows from the proof of Lemma 3 ,

By Lemmas 3 and $4, \Lambda$ is homeomorphic to Thurston's geodesic lamination on a surface of genus $g \geq 2$; cf Thurston [13]. To finish the proof of theorem, one needs "blow-down" $\Lambda$ to a measured foliation $\mathcal{F}$. The required interval exchange transformation $\varphi$ is the "mapping of first return" on a global transversal to $\mathcal{F}$. By the construction, $\varphi$ is minimal and has $n=2 g+m-1$ intervals of continuity.

Theorem 1 is proven.

\section{Criterion of strict ergodicity for the inter- val exchange transformations}

In general, group $\left(K_{0}, K_{0}^{+},[1]\right)$ may be not totally ordered. The total order happens if and only if positive cone $K_{0}^{+}$is bounded by unique "hyperplane" in the "space" $K_{0}$. There exists up to $(n-2)$ hyperplanes in a group $K_{0}$ of rank $n$ which constitute a boundary of $K_{0}^{+}$; cf Goodearl [4], p.217.

A state is a homomorphism $f$ from $\left(K_{0}, K_{0}^{+},[1]\right)$ to $\mathbb{R}$ such that $f\left(K_{0}^{+}\right) \subset$ $\mathbb{R}_{+}$and $f([1])=1$. The space of states $S_{\bullet}$ is dual to the linear space $K_{0}^{+}$. 
From this point of view, "hyperplanes" correspond to linearly independent "vectors" of the space $S_{\bullet}$. A total order is equivalent to the requirements $\operatorname{dim} S_{\bullet}=1$ and absence of "infinitesimals", cf Effros [2] p.26.

Let $\varphi$ be an interval exchange transformation built upon $\left(K_{0}, K_{0}^{+},[1]\right)$. Invariant measures of $\varphi$ form a vector space relatively sums and multiplication of measures by positive reals. This vector space is isomorphic to $S_{\bullet}$. The requirement $\varphi$ to be strictly (uniquely) ergodic is equivalent to the claim $S$. be one-dimensional. Strict ergodicity of the interval exchange transformations has been a challenging problem in the area for years. (Find a working criterion to determine whether given $\varphi$ is strictly ergodic.)

This saga started in 1975 when examples of interval exchange transformations with two and three invariant ergodic measures became known due to Keynes and Newton. Keane made an assumption that the "majority" of transformations $\varphi$ are strictly ergodic. This assumption was turned to a theorem independently by Masur and Veech who used for this purpose the Teichmüller theory and topological dynamics, respectively. The proof of Keane's conjecture based on Theorem 1 is given in Section 2.3.

In this section we establish strict ergodicity for a class of interval exchange transformations which we call "stationary". The name comes from theory of ordered abelian groups, because such transformations have stationary Bratteli diagrams; cf Effros [2]. Foliations that correspond to such transformations are known as pseudo-Anosov or foliations whose leaves are 1dimensional basic sets of the pseudo-Anosov homeomorphisms of a compact surface.

Definition 2 Let $\varphi=\varphi(\lambda, \pi, \varepsilon)$ be an interval exchange transformation whose Bratteli diagram is given by the infinite sequence of multiplicity matrices

$$
\left\{P_{Y_{1}}, P_{Y_{2}}, P_{Y_{3}}, \ldots\right\} \text {. }
$$

If the set (5) can be divided into the blocks $B_{k}=\left\{P_{Y_{n}}, P_{Y_{n+1}}, \ldots, P_{Y_{n+k}}\right\}$ such that $P_{Y_{n}} P_{Y_{n+1}} \ldots P_{Y_{n+k}}=P$, then $\varphi$ is called stationary. In particular, $\varphi$ is stationary if $P_{Y_{1}}=P_{Y_{2}}=P_{Y_{3}}=\ldots=P$.

Theorem 2 Every stationary interval exchange transformation $\varphi$ is strictly ergodic.

Proof. The proof is based on the Perron-Frobenius Theorem. A dual (projective) limit

$$
\left(\mathbb{R}^{n}\right)^{*} \stackrel{\mathrm{P}_{\mathrm{Y}_{1}}}{\longrightarrow}\left(\mathbb{R}^{n}\right)^{*} \stackrel{\mathrm{P}_{\mathrm{Y}_{2}}}{\longrightarrow}\left(\mathbb{R}^{n}\right)^{*} \stackrel{\mathrm{P}_{\mathrm{Y}_{3}}}{\longrightarrow} \ldots
$$


consists of operators $P_{Y_{i}}$ acting on the dual space $\left(\mathbb{R}^{n}\right)^{*}$ to $\mathbb{Z}^{n} \subset \mathbb{R}^{n}$. (In other words, we identify the space of positive homomorphisms $\mathbb{Z}^{n} \rightarrow \mathbb{R}$ and the space of linear functionals $\mathbb{R}^{n} \rightarrow \mathbb{R}$.) The diagram (6) converges to the state space $S_{\bullet}$ of dimension group $\left(K_{0}, K_{0}^{+},[1]\right)$. When $P_{Y_{i}}=P$, where $P$ is a matrix with strictly positive entries, or can be reduced to this case, then there exists a maximal simple eigenvalue $\lambda>0$ of matrix $P$ (PerronFrobenius Theorem). The eigenvector $x_{\lambda}$ defines a 1 -dimensional $P$-invariant subspace of $\left(\mathbb{R}^{n}\right)^{*}$ lying in the limit of diagram (6) and which is identified with $S_{\bullet}$. Let us formalize this idea.

For $1 \leq i \leq n$ denote by $e_{i}$ and $e_{i}^{*}$ the vectors of canonical bases in the vector space $\mathbb{R}^{n}$ and the dual space $\left(\mathbb{R}^{n}\right)^{*}$. By $\left(\alpha_{1}, \ldots, \alpha_{n}\right)$ and $\left(\alpha_{1}^{*}, \ldots, \alpha_{n}^{*}\right)$ we denote vectors in $\mathbb{R}^{n}$ and $\left(\mathbb{R}^{n}\right)^{*}$. $\left(\mathbb{R}^{n}\right)^{+}$and $\left(\mathbb{R}^{n}\right)^{*+}$ are collections of vectors whose coordinates are $\alpha_{i} \geq 0$ and $\alpha_{i}^{*} \geq 0$, respectively. The same notation $\left(\mathbb{Z}^{n}\right)^{+}$is reserved for the integer vectors of $\mathbb{R}^{n}$. By $\Delta_{0} \subset\left(\mathbb{R}^{n}\right)^{*}$ we understand n-dimensional simplex spanned by the vectors $0, e_{1}^{*}, \ldots, e_{n}^{*}$. To each linear mapping $\phi: \mathbb{R}^{n} \rightarrow \mathbb{R}^{n}$ one associates a dual mapping $\phi^{*}:\left(\mathbb{R}^{n}\right)^{*} \rightarrow\left(\mathbb{R}^{n}\right)^{*}$.

Denote by $P$ an dimension group corresponding to the limit

$$
P \cong \lim _{k \rightarrow \infty} P_{k}
$$

where $P_{k}$ are ordered groups whose positive cone is defined to be an inverse of $k$-th iteration of the set $\left(\mathbb{Z}^{n}\right)^{+}$under the automorphism $\phi$ :

$$
P_{k}^{+}=\phi^{-k}\left[\left(\mathbb{Z}^{n}\right)^{+}\right] .
$$

The set $\Delta_{0}$ has been introduced earlier. For $k=1, \ldots, \infty$ we let

$$
\Delta_{k}=S_{\bullet}\left(P_{k}\right),
$$

which is a state space of the group $P_{k}$. Define $\Delta_{k}$ to be a simplex spanned by the vectors $0, J_{1}(k), \ldots, J_{n}(k)$, where

$$
J_{1}(k)=\frac{X_{1}(k)}{\left\|X_{1}(k)\right\|}, \quad \ldots \quad, J_{n}(k)=\frac{X_{n}(k)}{\left\|X_{n}(k)\right\|},
$$

and

$$
X_{1}(k)=\phi^{k}\left(e_{1}^{*}\right), \quad \cdots \quad, X_{n}(k)=\phi^{k}\left(e_{n}^{*}\right) .
$$

It is evident that

$$
\Delta_{0} \supseteq \Delta_{1} \supseteq \Delta_{2} \supseteq \ldots \supseteq \Delta_{\infty}
$$


where

$$
\Delta_{\infty}=\bigcap_{k=1}^{\infty} \Delta_{k}
$$

A recurrent formula linking $X_{i}(k-1)$ and $X_{i}(k)$ is given by the equation:

$$
X_{i}(k)=\sum_{j=1}^{n} p_{i j} X_{j}(k-1),
$$

where $p_{i j}$ are the entries of matrix of "partial multiplicities" $P_{y}$.

Note that simplex $\Delta_{\infty}$ has dimension $r \leq n$. The original problem of calculating the state space $S_{\bullet}$ is reduced to calculation of the asymptotic simplex $\Delta_{\infty}$ whose spanning vectors are linked by equation (14). We shall see that $\Delta_{\infty}$ can be completely calculated under hypothesis of Theorem 2 . The following lemma is basic.

Lemma 5 (Perron-Frobenius) A strictly positive $n \times n$ matrix $P=\left(p_{i j}\right)$ always has a real and positive eigenvalue $\lambda$ which is a simple root of the characteristic equation and exceeds the moduli of all the other characteristic values. To this maximal eigenvalue $\lambda$ there corresponds an eigenvector $x_{\lambda}=$ $\left(x_{\lambda}^{1}, \ldots, x_{\lambda}^{n}\right)$ with positive coordinates $x_{\lambda}^{i}>0, i=1, \ldots, n .3$

Proof of lemma. Let $x=\left(x_{1}, x_{2}, \ldots, x_{n}\right)$ be a fixed vector. A function

$$
r_{x}=\min _{1 \leq i \leq n} \frac{(P x)_{i}}{x_{i}}
$$

\footnotetext{
${ }^{3}$ In fact, there exists more general statement due to Frobenius which treats matrices with non-negative entries. Because of exceptional importance of this statement in understanding why the unique ergodicity may vanish, and also due to the clear connection of Frobenius theorem with the root systems of Coxeter-Dynkin, we give the formulation of this theorem in below.

Theorem (Frobenius) An irreducible non-negative $n \times n$ matrix $P=\left(p_{i j}\right)$ always has a positive eigenvalue $\lambda$ that is a simple root of the characteristic equation. The moduli of all the other eigenvalues do not exceed $\lambda$. To the maximal eigenvalue $\lambda$ there corresponds an eigenvector with positive coordinates.

Moreover, if $P$ has $r$ eigenvalues $\lambda_{0}=\lambda, \lambda_{1}, \ldots, \lambda_{r-1}$ of modulus $\lambda$, then these numbers are all distinct and are roots of the equation

$$
z^{r}-\lambda^{r}=0
$$

More generally: The whole spectrum $\lambda_{0}, \lambda_{1}, \ldots, \lambda_{n-1}$ of $P$, regarded as a system of points in the complex plane, goes over into itself under a rotation of the plane by the angle $2 \pi / r$.
} 
is introduced. We have $r_{x} \geq 0$ since

$$
(P x)_{i}=\sum_{j=1}^{n} p_{i j} x_{j},
$$

is a non-negative matrix. However, in the definition of minimum (15), the values of $i$ for which $x_{i}=0$ are excluded. The lemma follows from the variational principle for the continuous function $r_{x}$, which must assume a maximal value for some vector $x$ with non-negative coordinates.

Now we can finish the proof of main theorem. By strict positivity of matrix $P$ and Lemma 5, there is a positive maximal eigenvalue $\lambda$, whose eigenvector $x_{\lambda}$ has positive coordinates. Notice that

$$
\phi^{k}\left(x_{\lambda}\right)=(\lambda)^{k} x_{\lambda}
$$

so that the iterations of $\phi$ leave invariant a 1-dimensional linear subspace $\{\alpha\}$ spanned by $x_{\lambda}$. All other vectors in $\left(\mathbb{R}^{n}\right)^{*+}$ converge accordingly (14) to the subspace $\{\alpha\}$. We conclude that

$$
\Delta_{\infty}=\{\alpha\}
$$

which is one-dimensional. Theorem 2 is proved.

Remark 1 In the context of ordered abelian groups, Theorem 2 was known to Effros [2] and Elliott [3]. The main ingredients of proof can be traced in the work 14 of W. Veech.

If $r>1$ then $P$ can be put into the following cyclic normal form

$$
\left(\begin{array}{ccccc}
O & P_{12} & O & \ldots & O \\
O & O & P_{23} & \ldots & O \\
\vdots & & & & \vdots \\
O & O & O & \ldots & P_{r-1, r} \\
P_{r 1} & O & O & \ldots & O
\end{array}\right)
$$

where $P_{i j}$ are non-zero square blocks along the main diagonal and $O$ are zero square blocks elsewhere. 


\section{Masur-Veech Theorem}

Theorem of Masur and Veech is formulated in Section 5.2. There are two known proofs of this theorem, due to Masur [6] who used complex analysis and Teichmüller theory and Veech [15] who used methods of topological dynamics. In this section we suggest an independent proof using Theorem 1 and one lemma of Morse and Hedlund from symbolic dynamics; cf Morse and Hedlund [8].

Parametrization of $\left(K_{0}, K_{0}^{+},[1]\right)$. 4 4 Let $\mathbb{H}, \tau$ and $S$ be as in Definition 1 of Section 2. Without loss of generality we assume that $S$ is a unit semicircle in the complex plane $\mathbb{H}$. Consider a family $S_{t}$ of the unit semi-circles parametrized by real numbers equal to a "horizontal shift" of $S$ in $\mathbb{H}$. (In other words, $t$ is equal to the $x$-coordinate of the centre of unit circle $S_{t}$.) A family of dimension groups which are defined by "positive cones" $S_{t}$, we shall denote by $\left(P, P_{+}^{t},[u]\right)$. By results of Sections 1-3 every dimension group of form $\mathbb{Z}^{n}$ has a representative in $\left(P, P_{+}^{t},[u]\right)$ and every measured foliation (with fixed singularity data) arises in this way.

Theorem 3 Denote by $\mathcal{F}_{t}$ a family of measured foliations corresponding to $\left(P, P_{+}^{t},[u]\right)$ and by $t_{1} \sim t_{2}$ an equivalence relation on $\mathbb{R}$ identifying topologically equivalent foliations $\mathcal{F}_{t_{1}}$ and $\mathcal{F}_{t_{2}}$. If $X=\mathbb{R} / \sim$ is a topological space, then for a residual set of the second category in $X$ foliation $\mathcal{F}_{t}$ is strictly ergodic.

Proof. The idea is to apply Koebe-Morse coding to each geodesic $S \in S_{t}$. In this setting, $X$ becomes a space of symbolic sequences with the topology described in Section 2. It is not hard to see that strict ergodicity of individual geodesic $S$ is equivalent to "uniform approximation" of $S$ by periodic sequences of length $N$. (Using the terminology of Morse and Hedlund, such approximation property of a geodesic means that a transitivity index $\phi(N)$ tends to a covering index $\theta(N)$ of the geodesic as $N \rightarrow \infty$; cf Morse and Hedlund [8].) The same authors proved that $\lim _{\mathbb{N} \rightarrow \infty}$ inf $\frac{\phi(N)}{\theta(N)}=1$ for a residual set of the second category in $X$. Let us give the details of this construction.

Let $S$ be a geodesic in the complex plane $\mathbb{H}$ and

$$
\sigma_{1}, \sigma_{2}, \sigma_{3}, \ldots
$$

\footnotetext{
${ }^{4}$ The idea of such parametrization was communicated to the author by G. A. Elliott.
} 
the Koebe-Morse code of $S$ which we shall call a ray; cf Section 3. The ray $R$ is transitive if it contains a copy of each admissible block. (Block is shorthand for a finite sequence of symbols.) Function $\phi: \mathbb{N} \rightarrow \mathbb{N}$ of a transitive ray $R$ is called a transitivity index if the initial block of $R$ of the length $\phi(N)$ contains all admissible blocks of the length $N$ and there are no shorter initial subblocks with this property. Dropping the claim that block $B \subset R$ is initial gives us function $\theta: \mathbb{N} \rightarrow \mathbb{N}$ which is called a covering index of the recurrent ray $R$. These functions satisfy an obvious inequality:

$$
\phi(N) \geq \theta(N) .
$$

Lemma 6 (Morse-Hedlund) A set of rays whose transitivity index and covering index satisfy the condition

$$
\lim _{N \rightarrow \infty} \inf \frac{\phi(N)}{\theta(N)}=1
$$

is a residual set of the second category in the space $X$ of all infinite rays endowed with the topology described in Section 2.

Proof of lemma. For a complete proof see [8]. Denote by $Y$ the set of rays satisfying the condition of lemma. The following items will be proved consequently:

(i) $Y$ is not empty;

(ii) $Y$ is everywhere dense in $X$;

(iii) The complement of $Y$ is nowhere dense in $X$.

(i) Let $H(n)$ be a block of minimum length containing all admissible blocks of length $n$. For a growing sequence of integers $r_{0}, r_{1}, \ldots, r_{k-1}$ consider a block

$$
H\left(r_{0}\right) \sigma_{1} H\left(r_{1}\right) \sigma_{2} \ldots \sigma_{k-1} H\left(r_{k-1}\right) \sigma_{k}
$$

of length $m_{k}$. Let us choose $r_{k}$ sufficiently large so that

$$
\frac{\theta\left(r_{k}\right)+m_{k}}{\theta\left(r_{k}\right)}<1+\delta_{k}
$$

where $\delta_{k}$ is a vanishing positive real. The transitivity index of (19) satisfies the inequality

$$
\phi\left(r_{k}\right) \leq \theta\left(r_{k}\right)+m_{k} .
$$


By the construction, $\frac{m_{k}}{\theta\left(r_{k}\right)} \rightarrow 0$ as $k \rightarrow \infty$ so that (19) satisfies the condition of lemma.

(ii) Let $A$ be an arbitrary admissible block of length $k$ and $R \in Y$. For a suitably chosen $\sigma$ the ray

$$
R^{\prime}=A \sigma R
$$

is admissible. We have the following inequalities for $R$ and $R^{\prime}$ :

$$
\theta(N) \leq \phi^{\prime}(N) \leq \phi(N)+k+1
$$

where $\phi$ and $\phi^{\prime}$ are the transitivity indices of $R$ and $R^{\prime}$. The condition of lemma is satisfied and therefore $R^{\prime} \in Y$.

(iii) This item follows from (ii) and accurate construction of closed sets lying in the complement of $Y$; cf [8] for the details. This argument finishes the proof of Morse-Hedlund lemma.

Let $R$ be a transitive ray and $B_{1}, \ldots, B_{k}$ admissible blocks of length $N$. We say that $R$ is uniformly distributed relatively $B_{1}, \ldots, B_{k}$ if

$$
\phi(N)=k N
$$

(In other words, each admissible block appears in the initial block of $R$ with the "probability" 1/k.) In the geometric terms this means that geodesic $R$ is located at the same distance from periodic geodesics

$$
B_{1}, B_{1}, \ldots ; \quad B_{2}, B_{2}, \ldots ; \quad \ldots ; \quad B_{k}, B_{k}, \ldots
$$

Lemma 7 Suppose that $R$ is uniformly distributed relatively admissible blocks $B_{1}, \ldots, B_{k}$ for each integer $N>0$. Then

$$
\lim _{N \rightarrow \infty} \inf \frac{\phi(N)}{\theta(N)}=1
$$

Proof of lemma. This follows from the equality $\phi(N)=\theta(N)$.

To finish the proof of Theorem 3 it remains to notice that strict ergodicity of $R$ is equivalent to uniform distribution of periodic "blocks" in $R$ and apply Lemmas 6 and 7 . 


\section{Appendix}

\subsection{Interval exchange transformations}

Let $n \geq 2$ be a positive integer and let $\lambda=\left(\lambda_{1}, \ldots, \lambda_{n}\right)$ be a vector with positive components $\lambda_{i}$ such that $\lambda_{1}+\ldots+\lambda_{n}=1$. One sets

$$
\beta_{0}=0, \quad \beta_{i}=\sum_{j=1}^{i} \lambda_{j}, \quad v_{i}=\left[\beta_{i-1}, \beta_{i}\right) \subset[0,1] .
$$

Let $\pi$ be a permutation on the index set $N=\{1, \ldots, n\}$ and $\varepsilon=\left(\varepsilon_{1}, \ldots, \varepsilon_{n}\right)$ a vector with coordinates $\varepsilon_{i}= \pm 1, i \in N$. An interval exchange transformation is a mapping $\varphi(\lambda, \pi, \varepsilon):[0,1] \rightarrow[0,1]$ which acts by piecewise isometries

$$
\varphi(x)=\varepsilon_{i} x-\beta_{i-1}+\beta_{\pi(i)-1}^{\pi}, \quad x \in v_{i},
$$

where $\beta^{\pi}$ is a vector corresponding to $\lambda^{\pi}=\left(\lambda_{\pi^{-1}(1)}, \lambda_{\pi^{-1}(2)}, \ldots, \lambda_{\pi^{-1}(n)}\right)$. Mapping $\varphi$ preserves or reverses orientation of $v_{i}$ depending on the sign of $\varepsilon_{i}$. If $\varepsilon_{i}=1$ for all $i \in N$ then the interval exchange transformation is called oriented. Otherwise, the interval exchange transformation is said to have flips.

Interval exchange transformation is said to be irreducible if $\pi$ is an irreducible permutation. An irreducible interval exchange transformation $T$ is called irrational if the only rational relation between numbers $\lambda_{1}, \ldots, \lambda_{n}$ is given by the equality $\lambda_{1}+\ldots+\lambda_{n}=1$. Recall that measure $\mu$ on $[0,1]$ is called invariant, if $\mu(\varphi(A))=\mu(A)$ for any measurable subset $A \subseteq[0,1]$. The following theorem due to M. Keane ([5]) estimates the number of invariant measures.

Finiteness Theorem. Let $\varphi$ be an irrational interval exchange transformation of $n$ intervals. Then there are at most finitely many ergodic invariant measures whose number cannot exceed $n+2$.

In case of the interval exchange transformations without flips, there exists an estimate of the number of invariant ergodic measures due to Veech [15].

Veech Theorem Let $\varphi$ be an irrational interval exchange transformation without flips on $n \geq 2$ intevals. Then the number of invariant ergodic measures of $\varphi$ is less or equal to $\left[\frac{n}{2}\right]$, where [•] is integer part of the number. 


\subsection{Measured foliations}

Measured foliations are suspensions over the interval exchange transformations which preserve the ergodic measure on intervals and such that their singularity set consists of $p$-prong saddles, $p \geq 3$. Measured foliations can be defined via closed 1-forms which is more elegant way due to Hubbard, Masur and Thurston.

Definition 3 (Hubbard-Masur-Thurston) Let $M$ be a compact $C^{\infty}$ surface of genus $g>1$, without boundary. A measured foliation $\mathcal{F}$ on $M$ with singularities of order $k_{1}, \ldots, k_{n}$ at points $x_{1}, \ldots, x_{n}$ is given by an open cover $U_{i}$ of $M \backslash\left\{x_{1}, \ldots, x_{n}\right\}$ and non-vanishing $C^{\infty}$ real valued closed 1 -form $\phi_{i}$ on each $U_{i}$, such that

(i) $\phi_{i}= \pm \phi_{j}$ on $U_{i} \cap U_{j}$;

(ii) at each $x_{i}$ there is a local chart $(u, v): V \rightarrow \mathbb{R}^{2}$ such that for $z=u+i v$, $\phi_{i}=\operatorname{Im}\left(z^{k_{i} / 2} d z\right)$ on $V \cap U_{i}$, for some branch of $z^{k_{i} / 2}$ in $U_{i} \cap V$.

Pairs $\left(U_{i}, \phi_{i}\right)$ are called an atlas for $\mathcal{F}$.

As it follows from the definition, apart from the singular points, measured foliations look like a non singular volume preserving flows. In singularities, the substitution $z \mapsto r e^{i \psi}$ brings $\phi_{i}$, mentioned in (ii), to the form

$$
\phi_{i}=r^{\frac{k_{i}}{2}}\left[\sin \left(\frac{k_{i}}{2}+1\right) \psi d r+r \cos \left(\frac{k_{i}}{2}+1\right) \psi d \psi\right] .
$$

It can be readily established, that $\phi_{i}$ are closed differential 1-forms, that is $d \phi_{i}=0$ for all $k_{i} \geq 1$. To see what singularities are generated by the above formula, let us consider a vector field $v_{i}$, given by the system of differential equations

$$
\frac{d r}{d t}=-r \cos \left(\frac{k_{i}}{2}+1\right) \psi, \quad \frac{d \psi}{d t}=\sin \left(\frac{k_{i}}{2}+1\right) \psi
$$

Clearly, $v_{i}$ is tangent to a foliation given by the equation $\phi_{i}=0$. Our prior interest is to study the behavior of trajectories of $v_{i}$ in a narrow stripe $\Pi=\{(r, \psi) \mid-\varepsilon \leq r \leq \varepsilon, 0 \leq \psi \leq 2 \pi\}$. There are exactly $k_{i}+2$ equilibria $p_{n} \in \Pi$, which have the coordinates $\left(0, \frac{2 \pi n}{k_{i}+2}\right)$, where $n \in \mathbb{N}$ varies from 0 to $k_{i}+2$. The linerization of the vector field $v_{i}$ in these points yields

$$
A\left(p_{n}\right)=\left(\begin{array}{cc}
(-1)^{n+1} & 0 \\
0 & (-1)^{n}\left(\frac{k_{i}}{2}+1\right)
\end{array}\right)
$$


Therefore all $p_{n}$ are the saddle points. One maps the half-stripe $r \geq 0$ to the neighbourhood of the singular point $x_{i}$. Generally, a singular point $x_{i}$ of the order $k_{i}$ is a $\left(k_{i}+2\right)$-prong saddle of a measured foliation $\mathcal{F}$.

Let $M$ be a compact surface and $\mathcal{F}$ a measured foliation on $M$. By measure $\mu$ of $\mathcal{F}$ one understands a line element $\|\phi\|$ related with the point $x \in M$, induced in each $x \in U_{i}$ by $\left\|\phi_{i}(x)\right\|$. It measures a 'transversal length' of $\mathcal{F}$, since $\mu$ vanishes in direction tangent to the leaves of $\mathcal{F}$.

Take a cross-section to the measured foliation $\mathcal{F}$. $\mathcal{F}$ induces an interval exchange transformation $\varphi$ on this cross-section. Depending on orientability of $\mathcal{F}, \varphi$ may have flips. Flips are excluded if $\mathcal{F}$ is an orientable measured foliation (in this case $\mathcal{F}$ is given by orbits of a measure-preserving flow). For orientable measured foliations, an estimate of number of invariant ergodic measures is due to Sataev [12].

Sataev Theorem Let $n$ and $k$ be a pair of natural numbers, such that $n \geq k$ and let $M$ be a compact orientable surface of genus $n$. Then there exists a $C^{\infty}$ orientable measured foliation $\mathcal{F}$ on $M$ whose singularity set consists of 4-separatrix saddles and which has exactly $k$ invariant ergodic measures.

An important question arises when measured foliation has a unique invariant measure. It was conjectured by M. Keane and proved by H. Masur and W. Veech that 'almost all' measured foliations have a unique invariant measure, which is a multiple of Lebesgue measure.

Masur-Veech Theorem ([6],[15]) Suppose that family $\mathcal{F}_{t}$ of measured foliations is given by trajectories of a holomorphic quadratic differential $e^{i t} \phi$ on the surface $M$. Then for 'almost all' values of $t$ foliation $\mathcal{F}_{t}$ is strictly ergodic.

\section{$5.3 \mathcal{O}_{\lambda}$ as a crossed product $C^{*}$-algebra}

Lemma 8 Let $\varphi=\varphi(\lambda, \pi, \varepsilon)$ be an interval exchange transformation and $\lambda=\left(\lambda_{1}, \ldots, \lambda_{n}\right)$. Then $K_{0}\left(\mathcal{O}_{\lambda}\right)=\mathbb{Z}^{n}$ and $K_{1}\left(\mathcal{O}_{\lambda}\right)=\mathbb{Z}$.

Proof. Let $p_{1}, \ldots, p_{n}$ be the set of discontinuous points of the mapping $\varphi$. Denote by $\operatorname{Orb} \varphi=\left\{\varphi^{m}\left(p_{i}\right): 1 \leq i \leq n, n \in \mathbb{Z}\right\}$ a set of full orbits of these points. When $\varphi$ is irrational, the set $\operatorname{Orb} \varphi$ is a dense subset in $[0,1]$. We replace every point $x \in \operatorname{Orb} \varphi$ in the interior of $[0,1]$ by two points $x^{-}<x^{+}$ 
moving apart banks of the cut. The obtained set is a Cantor set denoted by $X$.

A mapping $\varphi: X \rightarrow X$ is defined to coincide with the initial interval exchange transformation on $[0,1] \backslash \operatorname{Orb} \varphi \subset X$ prolonged to a homeomorphism of $X$. Mapping $\varphi$ is a minimal homeomorphism of $X$, since there are no proper, closed, $\varphi$-invariant subsets of $X$ except the empty set. Thus, $\mathcal{O}_{\lambda}=C(X) \rtimes_{\varphi} \mathbb{Z}$ is a crossed product $C^{*}$-algebra, where $C(X)$ denotes a $C^{*}$ algebra of continuous complex-valued functions on $X$. The following diagram of Pimsner and Voiculescu consists of exact sequences:

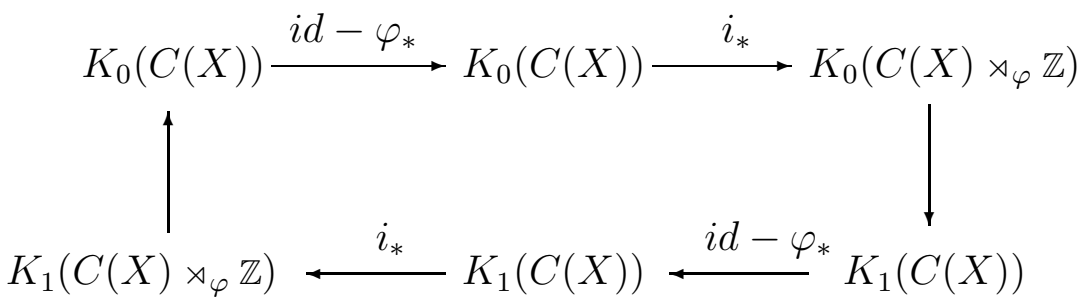

It was proved in [10] that $K_{0}(C(X)) \simeq \mathbb{Z}^{n}$ and $K_{1}(C(X)) \simeq 0$. To obtain the conclusion of Lemma 8 it remains to calculate all short exact sequences in the diagram of Pimsner and Voiculescu.

\subsection{Rotation numbers}

One of the striking invariants of algebra $\mathcal{O}_{\lambda}$ are rotation numbers associated to this algebra. In the dynamical context, rotation numbers are equal to "average inclination" of leaves of measured foliation relatively a coordinate system on $M_{n}$. (In fact, the original study of $\mathcal{O}_{\lambda}$ was motivated by possibility to introduce such numbers; if [9].) Rotation numbers for $\mathcal{O}_{\lambda}$ play the same role as real numbers $\theta$ for the irrational rotation algebra $A_{\theta}$.

Recall that the cone $K_{0}^{+} \subset \mathbb{H}$ is a limit of "rational" cones $P_{k}^{+} \subset \mathbb{H}$ :

$$
K_{0}^{+}=\lim _{k \rightarrow \infty} P_{k}^{+} .
$$

Each $P_{k}^{+}$is represented by a periodic geodesic $\gamma_{k}$. Suppose that $g_{k} \in G$ is an isometry which moves geodesic $\gamma_{k-1}$ to geodesic $\gamma_{k}$ and let

$$
g_{k}=\left(\begin{array}{cc}
a_{i} & b_{i} \\
c_{i} & d_{i}
\end{array}\right) \in P S L_{2}(\mathbb{Z})
$$


be an integral matrix with non-negative entries and determinant \pm 1 , corresponding to $g_{k}$. The continued fraction

$$
\theta_{\lambda}=\frac{a_{1}}{c_{1}}-\frac{c_{1}^{-2}}{\frac{d_{1}}{c_{1}}+\frac{a_{2}}{c_{2}}-\frac{c_{2}^{-2}}{\frac{d_{2}}{c_{2}}+\frac{a_{3}}{c_{3}}-\ldots}}
$$

converges to a real number $\theta_{\lambda}$ which is called a rotation number associated to algebra $\mathcal{O}_{\lambda}$. The importance of rotation numbers is stipulated by the following theorem.

Theorem Let $\mathcal{O}_{\lambda}$ and $\mathcal{O}_{\lambda}^{\prime}$ be two $C^{*}$-algebras whose rotation numbers are $\theta_{\lambda}$ and $\theta_{\lambda}^{\prime}$. Then $\mathcal{O}_{\lambda}$ is Morita equivalent to $\mathcal{O}_{\lambda}^{\prime}$ if and only if $\theta_{\lambda}$ and $\theta_{\lambda}^{\prime}$ are modular equivalent:

$$
\theta_{\lambda}^{\prime}=\frac{a \theta_{\lambda}+b}{c \theta_{\lambda}+d}, \quad a, b, c, d \in \mathbb{Z}, \quad a d-b c= \pm 1 .
$$

Proof. This was proved in [9].

Corollary 1 Suppose that $\varphi$ is a stationary interval exchange transformation described in Section 3. Then the rotation number $\theta_{\lambda}$ is a quadratic surd (i.e. irrational root of a quadratic equation).

Proof. By the results of Section 3, the dimension group of $\mathcal{O}_{\lambda}$ is stationary and must correspond to a periodic continued fraction (i.e. $g_{1}=g_{2}=\ldots=$ Const). These fractions generate a field of quadratic algebraic numbers.

\section{Conclusions and open problems}

The criterion of strict ergodicity of Section 3 is highly constructive and can be used in practice to check whether given interval exchange transformation is strictly ergodic or not. (This can find applications in the theory of billiards in the rational polygons.) Of course, these conditions are only sufficient. The necessary conditions seem to be an open problem so far.

Another open problem is to relate the arithmetic of rotation numbers $\theta_{\lambda}$ with the number of invariant measures of the transformation $\varphi$. (In the case of strictly ergodic $\varphi$ the answer is given by Corollary 1,) 


\section{References}

[1] O. Bratteli, Inductive limits of finite dimensional $C^{*}$-algebras, Trans. Amer. Math. Soc. 171 (1972), 195-234.

[2] E. G. Effros, Dimensions and $C^{*}$-Algebras, in: Conf. Board of the Math. Sciences, Regional conference series in Math., No.46, AMS, 1981.

[3] G. A. Elliott, On totally ordered groups and $K_{0}$, Ring theory (Proc. Conf., Univ. Waterloo, Waterloo, 1978), pp. 1-49, Lecture Notes in Math., Vol. 734, Springer, Berlin, 1979.

[4] K. R. Goodearl, Partially Ordered Abelian Groups With Interpolation, Mathematical Surveys and Monographs, 20. American Mathematical Society, Providence, R.I., 1986. xxii+336 pp. ISBN: 0-8218-1520-2

[5] M. Keane, Interval exchange transformations, Math. Z. 141 (1975), $25-31$.

[6] H. Masur, Interval exchange transformations and measured foliations, Ann. of Math. (2) 115 (1982), no. 1, 169-200.

[7] M. Morse, Recurrent geodesics on a surface of negative curvature, Trans. Amer. Math. Soc. 22 (1921), 84-100.

[8] M. Morse and G. A. Hedlund, Symbolic dynamics, Amer. J. of Math. 60 (1938), 815-866.

[9] I. Nikolaev, Invariant of minimal flows coming from the $K_{0}$-group of a crossed product $C^{*}$-algebra, Ergodic Theory Dynam. Systems 20 (2000), 1449-1468.

[10] Ian F. Putnam, The $C^{*}$-algebras associated with minimal homeomorphisms of the Cantor set, Pacific J. Math. 136 (1989), no. 2, 329-353.

[11] Ian F. Putnam, $C^{*}$-algebras arising from interval exchange transformations, J. Operator Theory 27 (1992), no. 2, 231-250.

[12] E. A. Sataev, On the number of invariant measures for flows on orientable surfaces, Izv. Akad. Nauk SSSR Ser. Mat. 39 (1975), no.4, 860-878. 
[13] W. P. Thurston, The Geometry and Topology of Three-Manifolds, MSRI 1997, electronic edition of 1980 Princeton Univ. notes, available at http://www.msri.org/gt3m/; alternative reference: ThreeDimensional Geometry and Topology, ed. by Silvio Levy, vol.1, Princeton Univ. Press, 1997.

[14] W. A. Veech, Interval exchange transformations, J. Analyse Math. 33 (1978), 222-272.

[15] W. A. Veech, Gauss measures for transformations on the space of interval exchange maps, Ann. of Math. (2) 115 (1982), no. 1, 201-242. 